\title{
The Epidemiology and Effectiveness of Synchronized Cardioversion in a UK Prehospital Setting: A Retrospective Cross-Sectional Study
}

\author{
Alan Cowley, MSc, MCPara; $\odot$ Dan Cody, MSc, MCPara; Magnus Nelson, MBBS, FRCEM
}

\author{
South East Coast Ambulance Service NHS \\ Foundation Trust, Crawley, United Kingdom of \\ Great Britain and Northern Ireland \\ Correspondence: \\ Alan Cowley, MSc, MCPara \\ South East Coast Ambulance Service NHS \\ Foundation Trust \\ Nexus House, Gatwick Road \\ Crawley, RH10 9BG, UK \\ E-mail: alan.cowley@secamb.nhs.uk
}

Conflicts of interest/funding: No funding was required for completion of this study and no conflict of interests are reported.

Keywords: cardioversion; critical care; prehospital; specialist paramedic
Abbreviations:
CCP: critical care paramedic
CCPBase: Critical Care Patient Database
CMP: Clinical Management Plan
NHS: National Health Service
PID: patient identifiable data
pVT: pulsed ventricular tachycardia
ROSC: return of spontaneous circulation
SECAmb: South East Coast Ambulance Service
NHS Foundation Trust
SVT: supra-ventricular tachycardia
UK: United Kingdom
USA: United States of America

Received: January 16, 2021

Revised: March 10, 2021

Accepted: March 18, 2021

\section{doi:10.1017/S1049023X21000546}

(C) The Author(s), 2021. Published by Cambridge University Press on behalf of the World Association for Disaster and Emergency Medicine. This is an Open Access article, distributed under the terms of the Creative Commons Attribution licence (http://creativecommons.org/ licenses/by/4.0/), which permits unrestricted re-use, distribution, and reproduction in any medium, provided the original work is properly cited.

\begin{abstract}
Background: Synchronized cardioversion is an internationally accepted standard therapy for unstable tachyarrhythmias, but it is conventionally an in-hospital physician-led intervention. Increasingly, it is being brought forward into the prehospital setting as part of a specialist paramedic scope of practice; however, very little literature exists regarding the epidemiology or efficacy in this setting.

Methods: All patients receiving cardioversion within a United Kingdom (UK) ambulance service were identified using an electronic database. The period of inclusion was March 1, 2017 through October 31, 2020. These data were then interrogated to provide demographic, physiological, and efficacy data, and then a sub-group was created to identify those who presented with a primary arrhythmia (as opposed to post-cardiac arrest).

Results: From a total of 93 patients, prehospital synchronized cardioversion successfully terminated the tachyarrhythmia in $96 \%$ of patients presenting with a primary arrhythmia ( $85 \%$ in the allcomers group) with a predominance towards males ( $82 \%$ of patients) and an average age of 67 years. Hypotension and reduced level of consciousness were the most commonly documented unstable features ( $84.4 \%$ and $44.4 \%)$.

Conclusion: Cardioversion within a paramedic-led service results in efficacy rates of $96 \%$ in patients presenting with a primary tachyarrhythmia. This is a similar efficacy rate to traditional doctor-led therapies. Demographic data show that males make up over $80 \%$ of the patient population, in keeping with previously published work across the spectrum of cardiac interventions.
\end{abstract}

Cowley A, Cody D, Nelson M. The epidemiology and effectiveness of synchronized cardioversion in a UK prehospital setting: a retrospective cross-sectional study. Prehosp Disaster Med. 2021;36(4):440-444.

\section{Introduction}

The use of synchronized cardioversion has been a primary therapy in the treatment of unstable tachyarrhythmias since the 1950s, but it is generally associated with an in-hospital or physician-led out-of-hospital scope of practice. Here presents a retrospective, crosssectional study of synchronized cardioversion by paramedics within a United Kingdom (UK) ambulance service.

Synchronized cardioversion is recognized as the primary therapy for unstable tachyarrhythmias by the Resuscitation Council UK (London, UK) Guidelines ${ }^{1}$ as well as many other international guidelines. Nevertheless, within the UK, it remains rare for this to be within the scope of practice of paramedic-led prehospital care teams, meaning that many patients presenting with an unstable tachyarrhythmia will have their treatment delayed. Little literature exists documenting the epidemiology and effectiveness of this treatment by paramedics.

South East Coast Ambulance Service National Health Service (NHS) Foundation Trust (SECAmb; Crawley, UK) has developed a workforce of specialist paramedics in critical care, comprised of experienced paramedics (minimum three years post-registration) who undertake postgraduate study, in-hospital placements, and work place based supervised practice. In addition, an enhanced governance system including case review and 24-hour remote consultant physician support have permitted the delivery of synchronized cardioversion within a Clinical Management Plan (CMP) for tachycardia that aligns with the Resuscitation Council Guidelines. There are ten critical care paramedic (CCP) teams distributed across the geographical area of the trust, operating 24 hours a day. Each CCP rotates through 


\begin{tabular}{|c|c|c|c|}
\hline Patients & All & Male & Female \\
\hline Gender, $\mathrm{n}$ (\% of total patients) & 94 & $77(81.9 \%)$ & $17(18.1 \%)$ \\
\hline Mean Age, years & 67.34 & 67.10 & 68.41 \\
\hline $\begin{array}{l}\text { Successful Synchronized } \\
\text { Cardioversion }\end{array}$ & $80(85.1 \%)$ & $64(83.1 \%)$ & $16(94.1 \%)$ \\
\hline $\begin{array}{l}\text { Average No. Attempts (successful } \\
\text { reversions) }\end{array}$ & 1.41 & 1.41 & 1.41 \\
\hline $\begin{array}{l}\text { Pulsed Ventricular Tachycardia } \\
\text { (pVT) }\end{array}$ & $69(73.4 \%)$ & $60(77.9 \%)$ & $9(52.9 \%)$ \\
\hline $\begin{array}{l}\text { Supra-Ventricular Tachycardia } \\
\text { (SVT) }\end{array}$ & $19(20.2 \%)$ & $12(15.6 \%)$ & $7(41.1 \%)$ \\
\hline Other & $6(6.4 \%)$ & $5(6.5 \%)$ & $1(5.9 \%)$ \\
\hline Chest Pain & $12(12.8 \%)$ & $7(9.1 \%)$ & $5(29.4 \%)$ \\
\hline Reduced Level of Consciousness & $58(61.7 \%)$ & $49(63.6 \%)$ & $9(52.9 \%)$ \\
\hline Other & $3(3.2 \%)$ & $3(3.9 \%)$ & $0(0.0 \%)$ \\
\hline
\end{tabular}

Table 1. Patient Characteristics - All Presentations

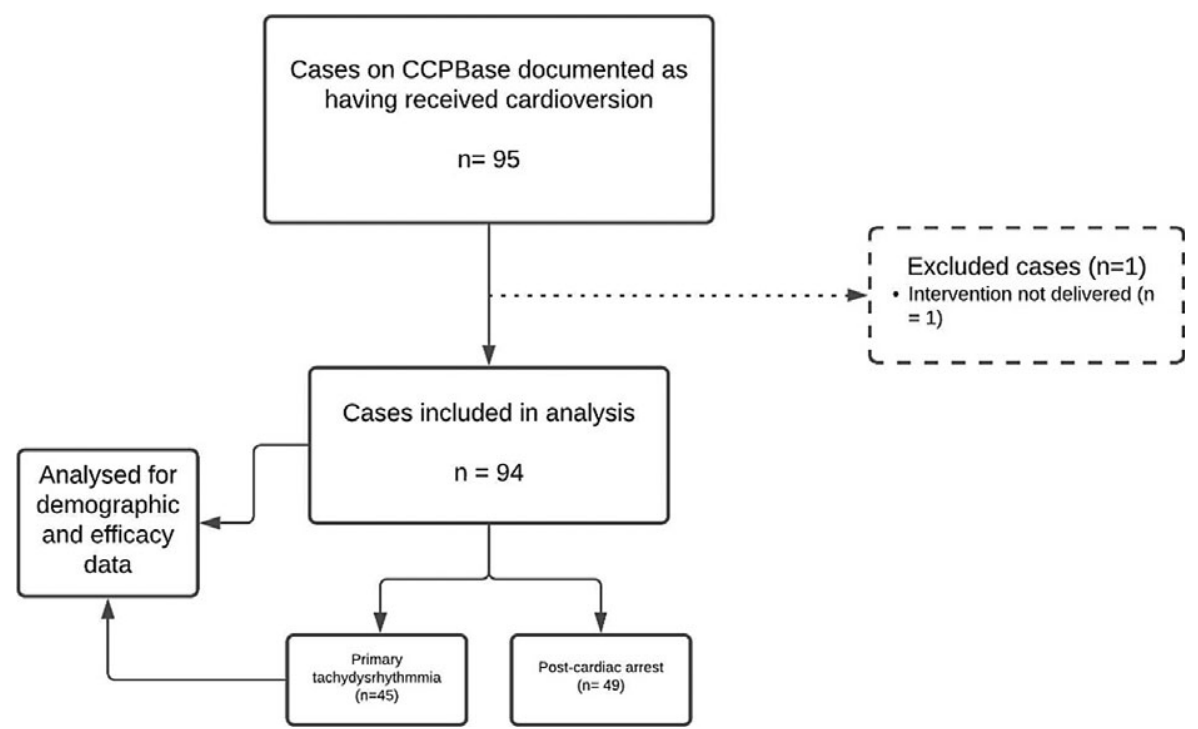

Figure 1. Data Filter Flow Chart.

Cowley @ 2021 Prehospital and Disaster Medicine Abbreviation: CCPBase, Critical Care Patient Database.

planned governance and skills assurance time to ensure appropriate oversight and safety and currency in practice. The rigor of this enhanced governance program facilitates the extended scope of practice and assurance of patient safety.

The CMP indicates synchronized cardioversion for any tachyarrhythmia where adverse features are present, including evidence of shock, syncope, myocardial ischemia, or heart failure. Three attempts at escalating energy levels are then permitted. If the rhythm fails to revert after three attempts, remote physician advice is sought to discuss further treatment options.
An extensive literature search of three major medical databases (CINAHL [EBSCO Information Services; Ipswich, Massachusetts USA], EMBASE [Elsevier; Amsterdam, Netherlands], and Medline [US National Library of Medicine, National Institutes of Health; Bethesda, Maryland USA]) revealed 77 matches, of which only four documented accounts of cardioversion by paramedics (or equivalent). The first was simply a list of all Emergency Medical Service interventions in the United States of America (USA). ${ }^{2}$ The second was an isolated case report from the USA, ${ }^{3}$ and the others were a small case series from Australia ${ }^{4}$ followed up by a comprehensive 


\begin{tabular}{|c|c|c|c|}
\hline Patients & All & Male & Female \\
\hline Gender, $\mathrm{n}$ (\% of total patients) & 45 & $35(77.8 \%)$ & $10(22.2 \%)$ \\
\hline Mean Age, years $[95 \% \mathrm{Cl}]$ & 68.98 [66.72-71.25] & 69.5 [67.35-71.65] & $67.1[60.25-73.96]$ \\
\hline Age Range & $28-96$ & $32-89$ & $28-96$ \\
\hline $\begin{array}{l}\text { Successful Synchronized } \\
\text { Cardioversion }\end{array}$ & $43(95.6 \%)$ & $34(97.1 \%)$ & $9(90.0 \%)$ \\
\hline $\begin{array}{l}\text { Average No. Attempts (successful } \\
\text { reversions) }\end{array}$ & 1.33 & 1.31 & 1.40 \\
\hline \multicolumn{4}{|l|}{ Presenting Rhythms } \\
\hline $\begin{array}{l}\text { Pulsed Ventricular Tachycardia } \\
\text { (pVT) }\end{array}$ & $29(64.4 \%)$ & $27(77.1 \%)$ & $2(20.0 \%)$ \\
\hline $\begin{array}{l}\text { Supra-Ventricular Tachycardia } \\
\text { (SVT) }\end{array}$ & $15(33.3 \%)$ & $8(22.9 \%)$ & $7(70.0 \%)$ \\
\hline Other & $1(2.2 \%)$ & $0(0.0 \%)$ & $1(10.0 \%)$ \\
\hline \multicolumn{4}{|l|}{$\begin{array}{l}\text { Presenting Features of Shock } \\
\text { (can be multiple) }\end{array}$} \\
\hline Hypotension & $38(84.4 \%)$ & $30(85.7 \%)$ & $8(80.0 \%)$ \\
\hline Altered Respiratory Rate & $5(11.1 \%)$ & $5(14.3 \%)$ & $0(0.0 \%)$ \\
\hline Chest Pain & $11(24.4 \%)$ & $6(17.1 \%)$ & $5(50.0 \%)$ \\
\hline Reduced Level of Consciousness & $20(44.4 \%)$ & $15(42.9 \%)$ & $5(50.0 \%)$ \\
\hline Other & $4(8.9 \%)$ & $4(11.4 \%)$ & $0(0.0 \%)$ \\
\hline Sedation Provided? & $45(100.0 \%)$ & $35(100.0 \%)$ & $10(100.0 \%)$ \\
\hline
\end{tabular}

Table 2. Patient Characteristics (Primary Tachyarrhythmia Group)

retrospective cohort study ${ }^{5}$ Both of these focused solely on supra-ventricular tachycardia (SVT). The last of these reported on efficacy, reporting a greater reversion rate than the previous literature. The generally accepted success rate is approximately $95 \%$ in pulsed ventricular tachycardia (pVT) and SVT. ${ }^{6}$

Separately, and most applicably, there was a study by Singer, et $\mathrm{al}^{7}$ that only looked at $\mathrm{pVT}$ but had similar aims to this study. It found a significant gender bias ( $84.1 \%$ male) with a mean age of 73.6 years (male) and 69.6 years (female). They also showed a $94 \%$ success rate in terminating the pVT. In a follow up, Fouche ${ }^{8}$ noted the under-utilization of this life-saving treatment, and so this study aims to widen the literature base regarding paramedic-led synchronized cardioversion. This will be achieved by retrospectively examining all cardioversions within the authoring trust and analyzing them for efficacy and demographic data.

\section{Setting}

The SECAmb is an urban, suburban, and rural NHS-funded ambulance service that broadly encompasses the counties of Sussex, Surrey, and Kent and receives nearly 862,000 calls each year. It employs almost over 4,000 staff, of which 84 (2.1\%) were operating as CCPs as of November 30, $2020 .^{9}$

\section{Methods}

A retrospective, cross-sectional descriptive study of all cases available from the SECAmb electronic Critical Care Patient Database (CCPBase [Medic One Systems Ltd.; London, England]), attended by SECAmb CCPs, from the first recorded use on the database (period March 1, 2017 through October 31, 2020) where synchronized cardioversion was recorded as administered, as per the CMP. Minimum recorded data for inclusion were age, sex, monitor upload (which provides electrocardiogram confirmation of rhythm and number of attempts), documentation of unstable feature(s), and documentation of result. Patients where recorded

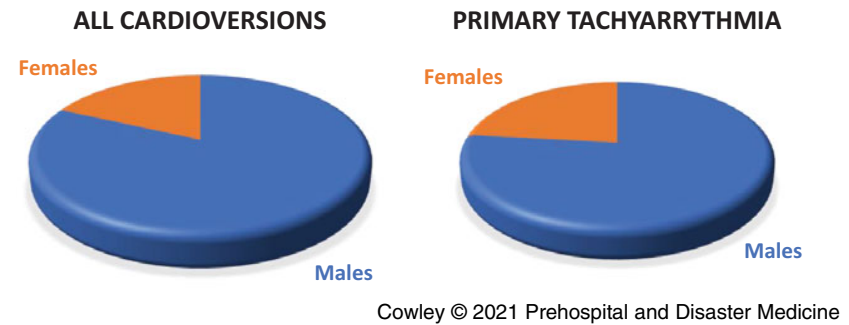

Figure 2. Gender Split.

data were incomplete were to be excluded (although there were no incomplete data).

Descriptive statistics were used to report patient characteristics, demographic, presenting rhythm, and basic therapy effectiveness. In an attempt to control confounding factors, a sub-group was then created to distinguish those patients with a primary tachyarrhythmia from those who had suffered a cardiac arrest and were cardioverted in their post-return of spontaneous circulation (ROSC) phase. This latter group of patients has multiple confounding factors due to their deranged physiology and mixed etiologies, and so it will be of far more benefit to the external validity of this study to examine the primary tachyarrhythmia patients only (Figure 1).

Stratton ${ }^{10}$ discusses the use of existing databases in research. In this case, the database used (CCPBase) is a secure, critical care database that the authoring trust uses as a supplement to the principal clinical record. Each record is entered by individual CCPs and was analyzed by $\mathrm{CCPs}$ within the leadership team. There was limited reliance on free text and data extraction was automated via an "export" function so there were no expected issues with interpretation or errors in data abstraction. Limited patient identifiable data (PID) are present in the database and are exported without any 


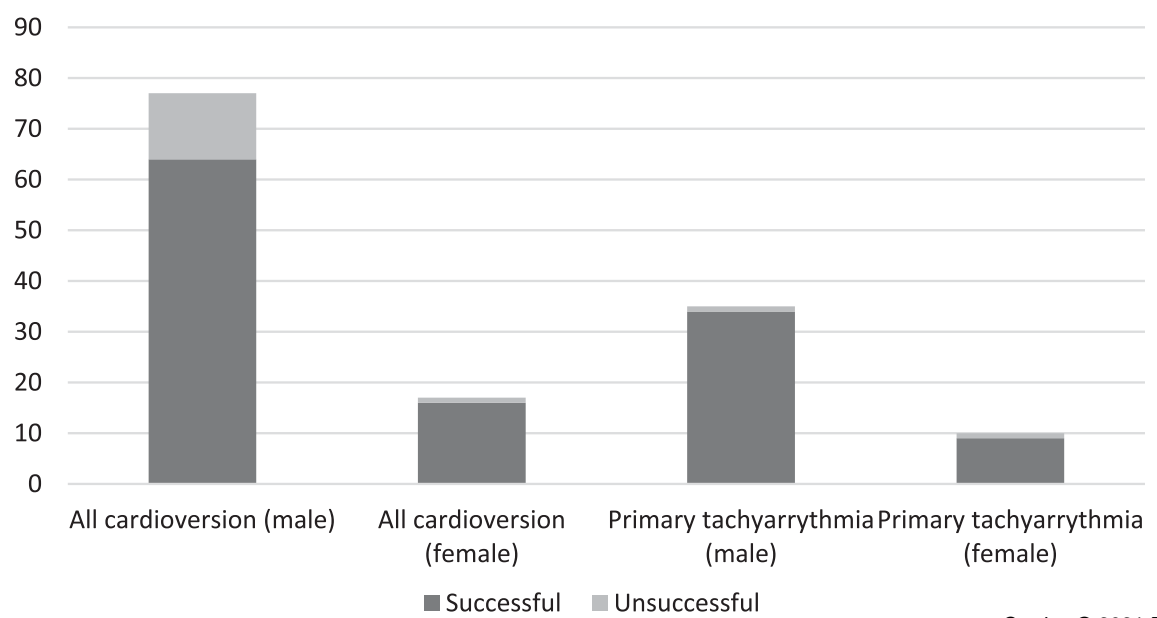

Figure 3. Cardioversion Success Rate by Gender.

Cowley @ 2021 Prehospital and Disaster Medicine

PID. Missing data could only occur where the clinician failed to enter a record in the first place - while this can't be eliminated, it is expected to include very low numbers of patients, if any.

The study obtained HRA Approval (IRAS ID: 290460) and received internal research and development approval. A full Data Protection Impact Analysis was performed and approved by the trust's information governance department.

\section{Results}

In total, 94 records were identified, of which only one was excluded due to therapy not having been delivered. There were no records that did not meet the minimum data set. Of these 94,45 presented with a primary tachyarrhythmia and 49 were in the post-ROSC phase following cardiac arrest. The full results are presented in Table 1. Table 2 displays the results for the group presenting with a primary tachyarrhythmia. This group was of primary interest as they had the most external validity and avoided the significant confounding factors associated with post-cardiac arrest physiology and mixed etiologies (Figure 2 and Figure 3).

Demographic data show an average age of 67 years [25-96], which varied little between genders or in the sub-group. Males were the predominant gender with almost $82 \%$ of the cardioversion attempts, reducing only slightly in the primary tachyarrhythmia group. In terms of successful attempts, the tachyarrhythmia group showed a $95 \%$ success rate at an average number of attempts of 1.4 (95\% CI of 0.17). Neither of these varied significantly between the sexes. The "all patients" group had a success rate of $85 \%$ across the genders with a similar average number of attempts. Pulsed ventricular tachycardia was the most common presenting rhythm in both groups, with hypotension and a reduced level of consciousness by far the most commonly documented unstable features.

\section{Discussion}

This study aims to widen the literature base regarding paramedicled cardioversion in the prehospital environment. Previous studies have taken place outside of the UK and have focused only on one of the two primary presenting rhythms and of varying scales. They described an unequal gender split and reversion rates of approximately $94 \%$, suggesting comparable efficacy rates to previously published rates in physician-led cardioversion.
The results of this study have demonstrated an efficacy rate of $95.6 \%$ in those patients presenting with primary tachyarrhythmias, which is very similar to those published previously. In addition, it has continued to demonstrate a clear predominance for males. The exact reasons for this are beyond the scope of this study, but this type of gender split is well-documented in various forms of cardiac intervention and is unlikely to be related to medical predisposition alone. Further studies into this wider subject, as well as larger studies to solidify cardioversion into paramedic prehospital practice, would be worthwhile.

The study is deemed to have good external validity and can likely be generalized to other paramedic-led programs. The authoring trust represents a good cross-section of the UK population, incorporating a variety of age groups, rural/urban populations, and economic prosperity. All primary tachyarrhythmias received sedation prior to intervention, so it would be more difficult to transfer the findings to programs without sedation capability. Confounding bias does exist in the "all patients" group and so the "primary tachyarrhythmia" group is the more externally valid.

\section{Limitations}

General limitations are confined to the confounding factors described within the "all patients" group and the somewhat limited numbers used in the calculations. The study relied on full documentation of the cardioversion attempt, and so it is not possible to ascertain if any other patients were cardioverted but not entered on to the database, and therefore not included in the study.

\section{Conclusion}

The study showed an $85 \%$ cardioversion cardiac rhythm conversion rate across all patients studied and a $95 \%$ cardiac conversion rate for the primary tachyarrhythmia sub-group. The average number of attempts was 1.4. These paramedic-led cardioversion efficacy rates are no different to those reported in the literature regarding doctorled attempts, and they also mirror the gender bias seen widely throughout the literature on cardiac intervention. While the safety profile was not an intended outcome of the study, no adverse events were reported during the cardioversion attempts. 


\section{References}

1. Pitcher J, Nolan D. Guidelines: Peri-Arrest Arrhythmias. London, United Kingdom: Resuscitation Council UK; 2015.

2. Carlson JN, Karns C, Mann NC, et al. Procedures performed by Emergency Medical Services in the United States. Prehosp Emerg Care. 2016;20(1):15-21.

3. Goss J. Complex cardioversion. JEMS. 2007;32(1):30.

4. Smith G, Taylor D, Morgans A, Cameron P. Prehospital synchronized electrical cardioversion of a poorly perfused SVT patient by paramedics. Prehosp Disaster Med. 2013;28(3):301-304.

5. Smith G, Taylor D, Morgans A, Cameron P. Prehospital management of supraventricular tachycardia in Victoria, Australia: epidemiology and effectiveness of therapies. Emerg Med Australas. 2014;26(4):350-355.
6. Trohman RG, Parillo JE. Direct current cardioversion: indications, techniques, and recent advances. Crit Care Med. 2000;28(10 Suppl):N170-173.

7. Singer S, Smith G, Stewart S, Stephenson M. Epidemiology and effectiveness of paramedic management of unstable pulsatile VT: preliminary findings. Australas $J$ Paramedicine. 2014;6(11):34.

8. Fouche P. Underutilization of synchronized cardioversion: Letter to the Editor. Emerg Med Australas. 2015;27(3):273-274.

9. South East Coast Ambulance Service NHS Foundation Trust. About Us. www. secamb.nhs.uk. Accessed November 24, 2020.

10. Stratton SJ. Using pre-existing databases for prehospital and disaster research. Prehosp Disaster Med. 2015;30(10):1-3. 